\title{
COHERENT TUNE SHIFTS IN THE BOOSTER
}

BOOSTER TECHNICAL NOTE

NO. 227

K. Zeno

September 6, 1995

ALTERNATING GRADIENT SYNCHROTRON DEPARTMENT

BROOKHAVEN NATIONAL LABORATORY

UPTON, NEW YORK 11973 


\title{
COHERENT TUNE SHIFTS IN THE BOOSTER
}

\author{
K. ZENO
}

September 6, 1995

\section{INTRODUCTION:}

This note attempts to measure the coherent tune shift in the Booster during standard high intensity running conditions. The measurements were motivated by the expectation that the tunes will differ considerably from what the BoosterTuneControl application indicates due to the effects of space charge. However, the aim of these measurements, and the analysis that follows, is only to determine the coherent tune shift, and compare it against a model.

When the beam is kicked as a whole the tune that is measured is not affected directly by the repulsive forces between the charged particles within the beam. It is the image charges and currents on the vacuum chamber and image currents on the pole faces of the dipole magnets which cause the beam to experience a tune shift. Therefore, little is learned directly about the tune shifts experienced by individual particles (the incoherent tune shift and spread).

It is assumed for the purpose of simplifying the analysis of the data that the tune predicted by the BoosterTuneControl application is correct for a beam in which space charge effects are negligible. This application estimates the tune by looking at the "trim" currents in the quadrupoles. It uses transfer functions between these "trim" currents and the tunes. These transfer functions were determined empirically at low intensity by W. van Asselt. They differ slightly from what the MAD model predicts. ${ }^{1}$ The agreement could have been verified by measuring the tunes at low intensity. The difference between measured and BoosterTuneControl application tunes is attributed to a shift in the coherent tune due to space charge effects. Expressions for the coherent tune shift exist for both planes. ${ }^{2}$ The tune shifts given by these expressions are compared with the 'measured' tune shifts. There is reasonable agreement between them.

An estimate of the incoherent tune spread was also made using much of the apparatus developed for the coherent tune shift analysis, the MAD model ${ }^{3}$, and a beam size measurement on MW006 from the Morning Numbers ${ }^{4}$. The details are given in the Appendix.

\section{METHOD:}

The tunes were measured using the BoosterTuneMeter program. For each plane, the tune was measured by kicking the beam in that plane, and performing an FFT on the position data obtained from a pick-up electrode in that plane. Early in the cycle, where the tunes in either 
plane were relatively far apart, the kicks were performed simultaneously. Later in the cycle, where the tunes were closer together, the kicks occured on different Booster pulses.

Measurements were taken at small time intervals in the early part of the cycle, where the beam survival is most sensitive to the tune. Later in the cycle, the time intervals between measurements were longer. The tunes during the last 10 milliseconds or so of the cycle were not measured.

The kick size was reduced from $14 \mathrm{kV}$ to $5 \mathrm{kV}$ when a possible relationship between the measured tune and the kick size was observed. This was noticed after the first eight sets of data were taken. The time interval covered by this data was BT0+9.15ms to $9.70 \mathrm{~ms}$. Subsequently, the kick amplitude was scaled roughly with momentum to obtain the cleanest measurement. Some beam loss may have occurred from the kick during the first eight sets of data.

Injection began at $9.07 \mathrm{~ms}$ from BT0. The fast chopper was sending the Booster 250 degree bunches for $500 \mu \mathrm{s}$. The intensity was not measured, but was typically $\sim 20 \mathrm{e} 12$ protons at extraction for this type of setup. From a current transformer trace at similar intensity, the intensity falls off from $25 \mathrm{e} 12$ to $20 \mathrm{e} 12$ within the first $5 \mathrm{~ms}$ and then remains essentially constant. $^{5}$

The Band III RF cavities were on during this study. This may have had an effect on the peak line charge density, which affects the tune. The peak line charge density could have been measured using the wall current monitor. The live magnet function and RF functions were used to model the theoretical coherent tune shift. 


\section{DATA:}

The data is shown in tabular form at the back of this note. Below are graphical representations of the tune data.

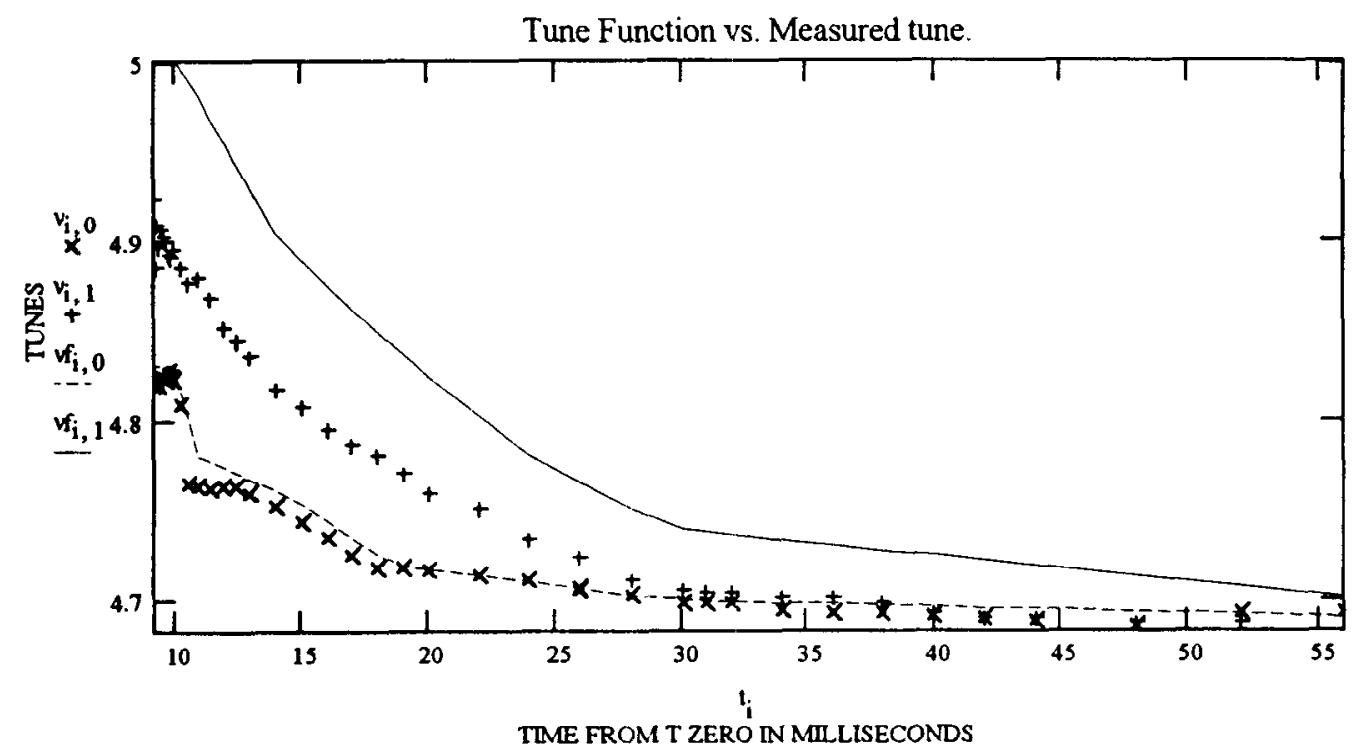

Graph 1: The tune functions as calculated in BoosterTuneControl together with the measured tunes.

+ 's are measured data points in the vertical.

$x$ 's are measured data points in the horizontal.

Solid line is BoosterTuneControl vertical tune.

Dashed line is BoosterTuneControl horizontal tune.

$\Delta v x_{i}=v_{i, 0}-v f_{i, 0} \quad$ These are the differences between the

$\Delta v y_{i}=v_{i, 1}-v_{i, 1} \quad$ measured and BoosterTuneControl tunes in each plane.

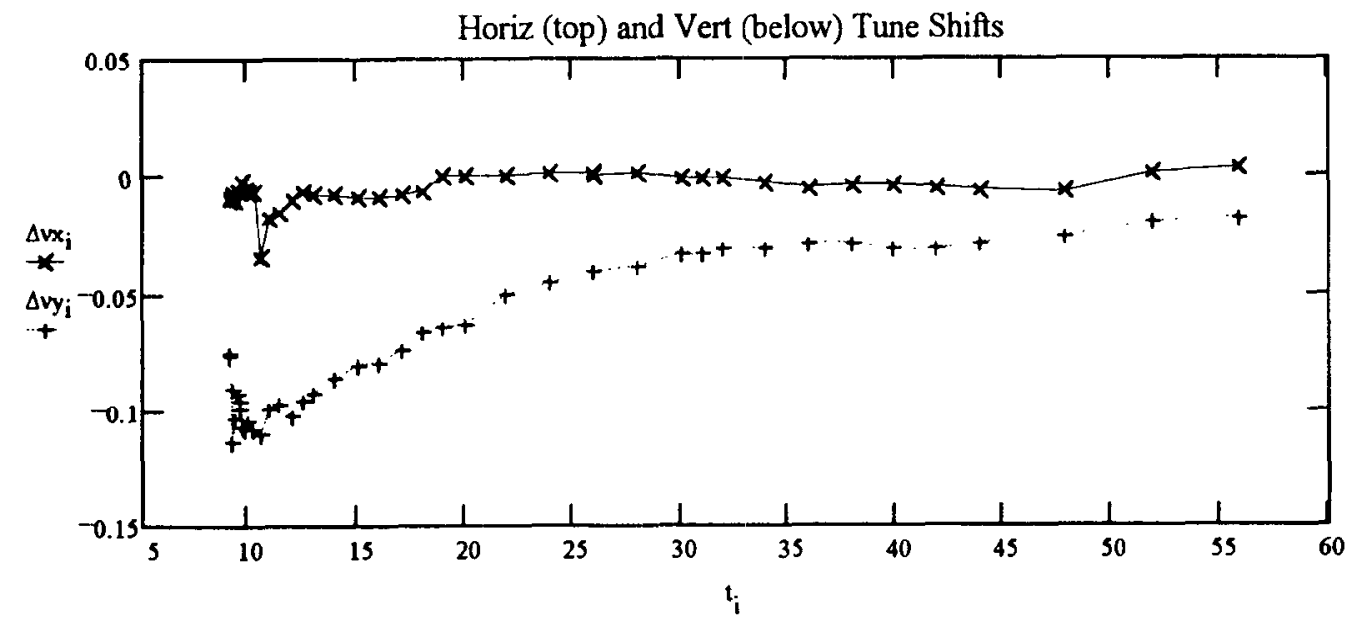

Graph 2: (Measured Tune)-(BoosterTuneControl tune) in both planes. 


\section{ANALYSIS:}

The analysis was done using Mathcad (calculation software on a PC). The analysis is presented as a Mathcad file.

\section{[1]. CONSTANTS:}

These constants are used in modeling the Coherent tune shift.

$\gamma_{\mathrm{tr}}:=4.88 \quad$ Transition $\gamma$.

$\mathrm{m}=0.9383 \quad$ Mass of proton $\left[\mathrm{GeV} / \mathrm{c}^{\wedge} 2\right]$.

$p=13.86557$ Booster bending radius $[\mathrm{m}]$.

$\mathrm{q}=1.602 \cdot 10^{-19}$ Charge of proton in [coul].

$\mathrm{R}=32.114 \quad$ Booster radius $[\mathrm{m}]$

c $=2.9979 \cdot 10^{8}$ speed of light $[\mathrm{m} / \mathrm{s}]$

$\mathrm{mp}:=938.259$ proton mass $\left[\mathrm{MeV} / \mathrm{c}^{\wedge} 2\right]$

me $=0.511 \quad$ electron mass $\left[\mathrm{MeV} / \mathrm{c}^{\wedge} 2\right]$

$i:=0,1.41 \quad$ Number of data points. The letter ' $i$ ' is used to index the data.

\section{[2]. PARAMETERS:}

These are parameters used in modeling the coherent tune shift.

[2.1]. Rough model of intensity variation during acceleration cycle $\left({ }^{*} 10^{\wedge} 13\right.$ charges $)$. Modeled after current transformer trace in HEP Startup Book III $(5 / 14 / 95)^{5}$.

$I_{i}=$ if $\left[i>18,2,2+\left(0.707 \cdot \frac{14-t_{i}}{4.85}\right)^{2}\right] \begin{aligned} & \text { Mathcad description of intensity model } \\ & \text { shown below. }\end{aligned}$

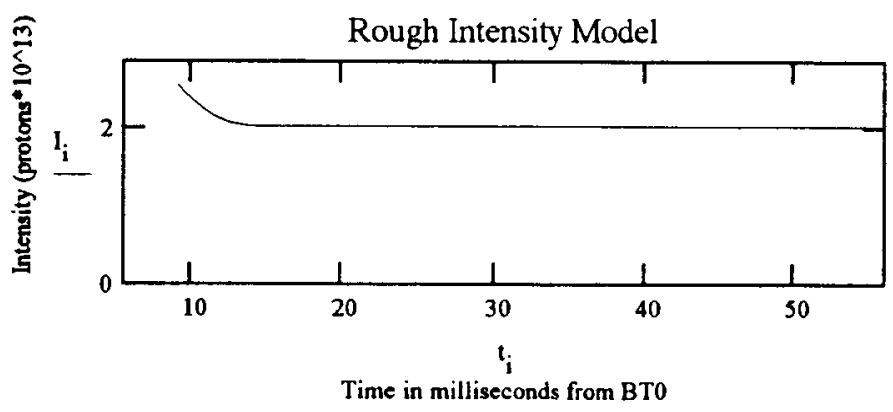

Graph 3: Rough model of the intensity variation through the Booster cycle.

$p_{i}=(0.000029979250) \cdot B_{i} \cdot \rho$ Momentum in $\mathrm{GeV} / \mathrm{c}$ 


$$
\begin{array}{ll}
\gamma_{i}=\sqrt{1+\frac{\left(p_{i}\right)^{2}}{m^{2}}} \quad \text { Lorentz factor. } & \beta_{i}=\sqrt{1-\frac{1}{\left(y_{i}\right)^{2}}} \\
\eta_{i}:=\frac{1}{\gamma_{t r}^{2}}-\frac{1}{\left(\gamma_{i}\right)^{2}} & \text { Slip factor } \\
f_{i}=\frac{\beta_{i} \cdot c}{2 \cdot \pi \cdot R} \quad \text { Revolution frequency. }
\end{array}
$$

[2.2]. The classical proton radius is used in the formulas for the coherent tune shifts. ${ }^{2,6}$

Calculating the Classical proton radius from the Classical electron radius.

$$
\begin{array}{ll}
\mathrm{r}_{\mathrm{e}}:=2.818 \cdot 10^{-15} & \text { Classical electron radius } \\
\mathrm{r}_{\mathrm{o}}:=\mathrm{r}_{\mathrm{e}} \mathrm{e} \cdot \frac{\mathrm{me}}{\mathrm{mp}} & \begin{array}{l}
\text { Ratio of masses multiplied by the Classical } \\
\text { electron radius gives the proton radius. }
\end{array} \\
\mathrm{r}_{\mathrm{o}}=1.535 \cdot 10^{-18} & \text { is the classical proton radius in meters. }
\end{array}
$$

\section{[2.3]. ESTIMATING THE PEAK LINE CHARGE DENSITY}

The peak line charge density is needed in the coherent tune shift formula. It could have been measured directly using the wall current monitor. However, this was not done, so an estimate is made.

Estimate the bunch width at each measurement time.

[2.3.1]. Find the point in the cycle where the bucket area is a minimum. Assume that the emittance is equal to the bucket area at this point.

[2.3.2]. Find the bunch width at this point by using the maximum phase extent equation. ${ }^{7}$

[2.3.3]. Find constant of proportionality $(K)$ in the equation, $A=\pi^{*} K^{*} U^{*} \Delta \phi$ at the point where the bucket area is a minimum. $A$ is the longitudinal emittance (constant), assumed equal to the bucke area. $\Delta \phi$ (the bunch width) was estimated in [2.3.2]. $\mathrm{W}=\mathrm{K}^{*} \mathrm{U}$ is the energy width of the bunch in phase space. $U$ is a function of the 'known' parameters: $p, R, V, \phi s, f$ and $\eta$ (see Weng and Mane, pg. 41).

[2.3.4]. Now that $K$ is known solve $A=\pi^{*} K^{*} U^{*} \Delta \phi$ for the bunch width and calculate it for each data point. 
point.

[2.3.5]. Convert $\Delta \phi$ into bunch width in meters.

[2.3.6]. Assume a parabolic shape for the bunch and estimate the peak line charge from the intensity and bunch width at each data point.

[2.3.1]. The RfBeamControl program gives a minimum bucket size of $7.5 \mathrm{eV}^{*} \mathrm{~s}$ near $52 \mathrm{~ms}$ (the 40 th data point). ${ }^{15}$

$$
\mathrm{A}=7.5 \quad \text { Bucket area in } \mathrm{eV}^{*} \mathrm{~s}
$$

[2.3.2]. The extreme phase extent equation is,

$$
\cos (\phi e)-\phi \cdot \sin (\phi s)=(\pi-\phi s) \cdot \sin (\phi s)-\cos (\phi s) \mathrm{v}
$$

It is derived from the synchronous phase which is calculated in the BoosterRfBeamControl program. фe is the 'extreme phase extent'.?

$\phi s r=\phi s \cdot d e g \quad$ Converts synchronous phase from degrees to radians.

The following finds the roots of the extreme phase extent equation for each measurement time. These roots are the extreme phases.

$\mathrm{j}:=0,1.25$ per $=3 \quad$ Guess for extreme phase extent early in the cycle.

$$
\text { фext }_{\mathrm{j}}=\operatorname{root}\left[\left(\cos (\phi e r)+\cos \left(\phi s r_{j}\right)\right)-\left[\left(\pi-\phi s r_{j}\right) \cdot \sin \left(\phi s r_{j}\right)+\phi e r \cdot \sin \left(\phi s r_{j}\right)\right], \phi e r\right]
$$

$j=26,27 . .41$ er $=1.2$ Guess for extreme phase extent later in the cycle.

$$
\text { pext }_{\mathrm{j}}=\operatorname{root}\left[\left(\cos (\phi e r)+\cos \left(\phi \mathrm{sr}_{\mathrm{j}}\right)\right)-\left[\left(\pi-\phi \mathrm{sr}_{\mathrm{j}}\right) \cdot \sin \left(\phi \mathrm{sr}_{\mathrm{j}}\right)+\phi \mathrm{\phi r} \cdot \sin \left(\phi \mathrm{sr}_{\mathrm{j}}\right)\right], \phi e r\right]
$$

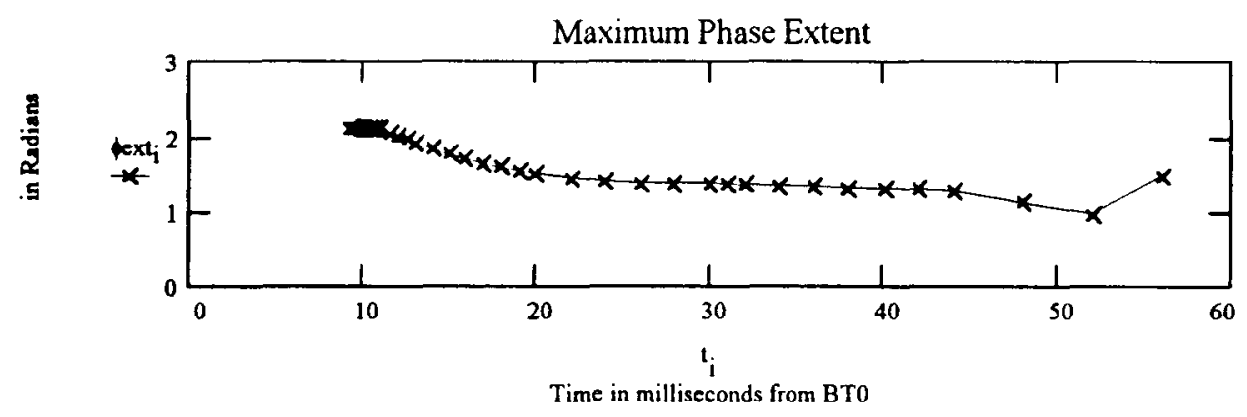

Graph 4: Maximum phase extent at measurement points.

The minimum bucket area occurs near the 40th data point. The extreme phase has a minimum here as well.

$$
\text { dext }_{40}=0.971 \text { radians }
$$




\section{[2.3.3]. Find $\mathrm{K}$ ? $^{\text {? }}$}

$\mathrm{K}=\frac{\operatorname{ext}{ }_{40} \cdot \pi}{\mathrm{A}} \cdot\left(\frac{\mathrm{P}_{40} \cdot \mathrm{R} \cdot \mathrm{V}_{40} \cdot 10^{3} \cdot \cos \left(\phi \mathrm{\phi s}_{40} \cdot \mathrm{deg}\right)}{\eta_{40} \cdot 2 \cdot \pi \cdot \mathrm{f}_{40}}\right)^{\frac{1}{4}} \quad$ leads to $\quad \mathrm{K}=0.548$

[2.3.4]. Calculate the bunch width for each point.

$$
\Delta \phi_{i}:=\frac{A}{\pi} \cdot K \cdot\left(\frac{\eta_{i} \cdot 2 \cdot \pi \cdot f_{i}}{p_{i} \cdot R \cdot V_{i} \cdot 10^{3} \cdot \cos \left(\phi s_{i} \cdot d e g\right)}\right)^{\frac{1}{4}}
$$

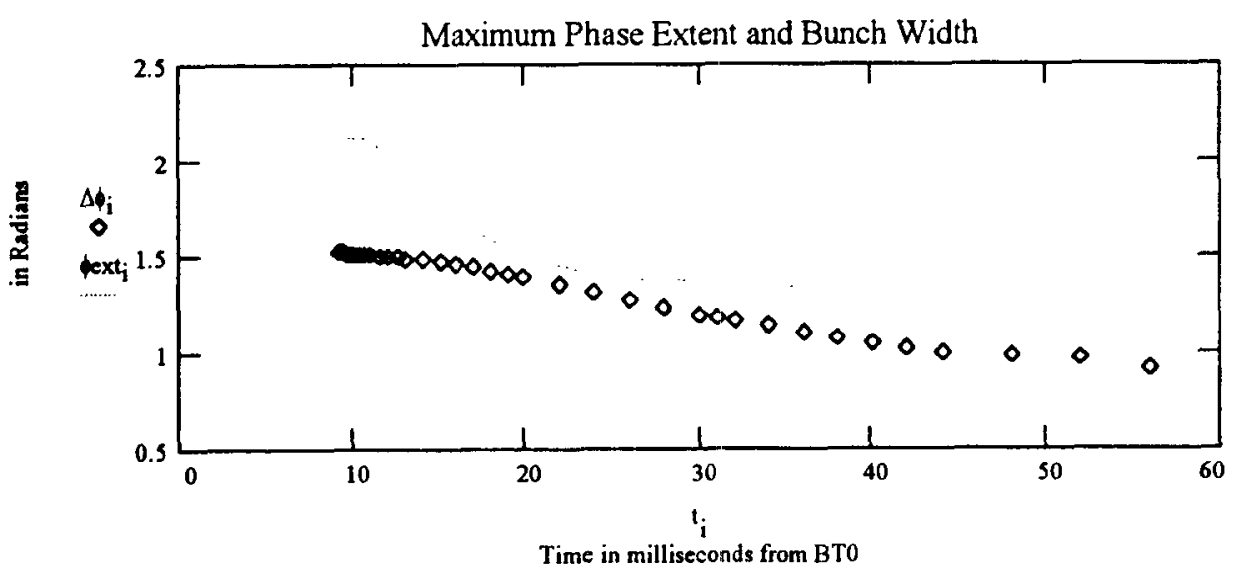

Graph 5: Maximum phase extent (dotted line) and estimated bunch width (in radians).

[2.3.5]. Convert bunch width to meters.

$$
b_{i}=\frac{\Delta \phi_{i}}{4 \cdot \pi} \cdot 2 \cdot \pi \cdot R \quad \text { Half-width of bunches in meters. }
$$

[2.3.6]. Estimate peak line charge density assuming parabolic distribution.

$$
\text { Int }=\int_{-b}^{b} p \lambda \cdot\left[1-\left(\frac{x}{b}\right)^{2}\right] d x \quad \begin{aligned}
& \operatorname{In} t=\frac{4}{3} \cdot p \lambda \cdot b \\
& \operatorname{Int}=I_{i} \cdot 10^{13}
\end{aligned} \lambda_{i}=\frac{\frac{3}{8} \cdot I_{i} \cdot 10^{13}}{b_{i}} \quad \begin{aligned}
& \text { Solving for the peak } \\
& \text { line charge density. }
\end{aligned}
$$




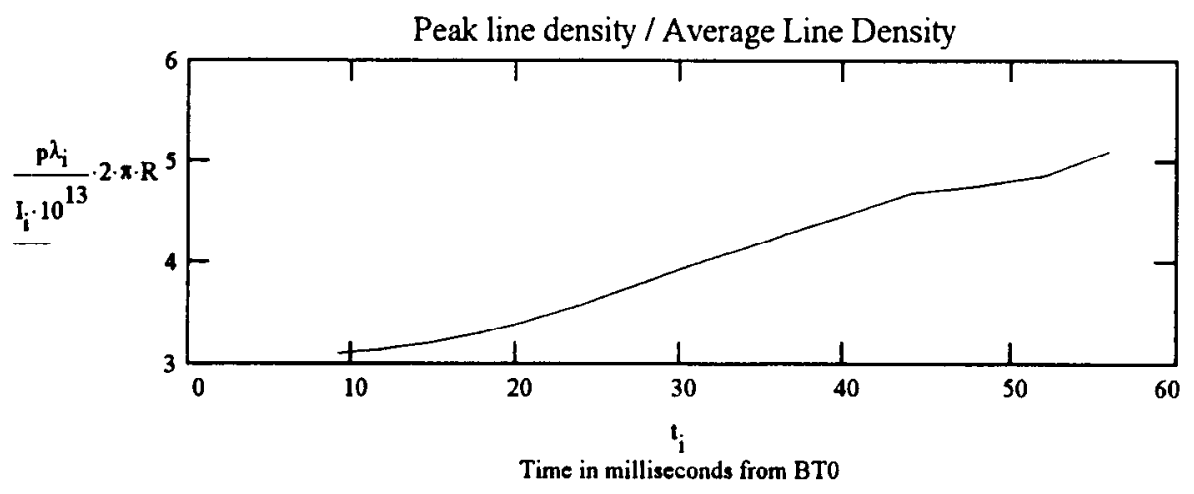

Graph 6: Ratio of estimated peak line charge density to average line charge density (reciprocal of bunching factor).

\section{[3].Calculating the Theoretical Tune Shift for Each Plane.}

\section{MORE CONSTANTS:}

$\mathrm{L}:=2.4 \quad$ Length of dipole magnet $[\mathrm{m}] .^{8}$

$\mathbf{n}:=36 \quad$ Number of dipole magnets in the ring. ${ }^{8}$

$1_{\text {dipole }}:=\mathrm{L} \cdot \mathrm{n} \quad 1_{\text {dipole }}=86.400 \quad$ Length of the ring filled with dipoles

$\mathrm{fr}:=\frac{1_{\text {dipole }}}{2 \cdot \pi \cdot \mathrm{R}} \quad$ Fraction of ring with dipole field $\mathrm{fr}=0.428$

$\mathbf{g}:=\frac{3.375}{2} \cdot 0.0254 \quad \begin{aligned} & \mathrm{g} \text { is the distance between dipole pole tips } / 2[\mathrm{~m}] . \\ & \text { Obtained from direct measurement }\end{aligned}$

$g=0.043$

$\lambda_{i}:=\frac{I_{i} \cdot 10^{13}}{2 \cdot \pi \cdot R} \quad$ Average line charge density.

$\mathbf{a}_{\mathrm{y}}=0.031$ These are the effective radii

for an elliptical vacuum chamber

$a_{x}=0.092$ and high frequency image fields $[\mathrm{m}] .^{2}$

$a_{\mathrm{dc}}:=0.0584$ This is the equivalent radius for an elliptical vacuum chamber and dc image fields $[\mathrm{m}]]^{2}$

$a_{0}:=0.076$ Radius of vacuum chamber outside dipoles $[\mathrm{m}] .^{8}$ 
There are several components of the tune shift. The skin depth of the vacuum chamber at the revolution frequency is much less than the thickness of the chamber walls. The fields outside the vacuum chamber are approximated as a function of the DC component of beam current. The magnetic field induced by this DC current is perpendicular to the pole tips of the dipoles and has a quadrupole moment. ${ }^{2,6}$

There is an AC component of the magnetic field caused by the changing electric field produced by the time-varying charge density of the beam. This magnetic field has the boundary condition that it is parallel to the surface of the vacuum chamber, since the electric field must be perpendicular to it. It also has a quadrupole moment. ${ }^{2,6}$

There is also an electric field caused by the image charges on the vacuum chamber which defocuses the beam in both planes. ${ }^{2,6}$

In general, the formulas can be written in the form²,

$$
\delta v=\frac{-\mathrm{r}_{0} \cdot R^{2}}{v \cdot \beta^{2} \cdot \gamma} \cdot\left(\frac{1}{\gamma^{2}} \cdot F+\beta^{2} \cdot G\right) \cdot
$$

Both functions $F$ and $G$ are dependent on the cross sectional shape of the vacuum chamber and the location and length of the dipole pole faces. Within the dipoles the cross section is approximated as elliptical $(70 \mathrm{~mm} \times 152 \mathrm{~mm})$, outside it is circular $(152 \mathrm{~mm} \times 152 \mathrm{~mm}){ }^{8} \mathrm{G}$ is also a function of the distance between the dipole pole faces.

For the vertical plane ${ }^{2}$,

For the horizontal plane ${ }^{2}$,

$$
\begin{aligned}
& \mathrm{Fy}_{i}:=\left[\frac{1}{\mathrm{a}_{0}^{2}} \cdot(1-\mathrm{fr})+\left(\frac{1}{\mathrm{a}_{\mathrm{y}}^{2}}\right) \cdot \mathrm{fr}\right] \cdot p \lambda_{i} \\
& \mathrm{~Gy}_{i}=\lambda_{i} \cdot\left(\frac{1}{\mathrm{adc}^{2}}+\frac{\pi^{2}}{12 \cdot \mathrm{g}^{2}}\right) \cdot \mathrm{fr}
\end{aligned}
$$$$
F x_{i}:=\left[\frac{1}{a_{0}^{2}} \cdot(1-f r)+\left(\frac{1}{a_{x}^{2}}\right) \cdot f r\right] \cdot p \lambda_{i}
$$$$
\mathrm{Gx}_{\mathrm{i}}=-\lambda_{\mathrm{i}} \cdot\left(\frac{1}{\mathrm{adc}^{2}}+\frac{\pi^{2}}{12 \cdot \mathrm{g}^{2}}\right) \cdot \mathrm{fr}
$$

The tune shifts for each plane are,

$$
\begin{aligned}
& \delta v y_{i}=\frac{-r_{0} \cdot R^{2}}{v_{i, i} \cdot\left(\beta_{i}\right)^{2} \cdot y_{i}} \cdot\left[\frac{1}{\left(\gamma_{i}\right)^{2}} \cdot F y_{i}+\left(\beta_{i}\right)^{2} \cdot G y_{i}\right] \\
& \delta v x_{i}:=\frac{-r_{0} \cdot R^{2}}{v_{i, 0} \cdot\left(\beta_{i}\right)^{2} \cdot y_{i}} \cdot\left(\frac{1}{\left(\gamma_{i}\right)^{2}} \cdot F x_{i}+\left(\beta_{i}\right)^{2} \cdot G x_{i}\right]
\end{aligned}
$$




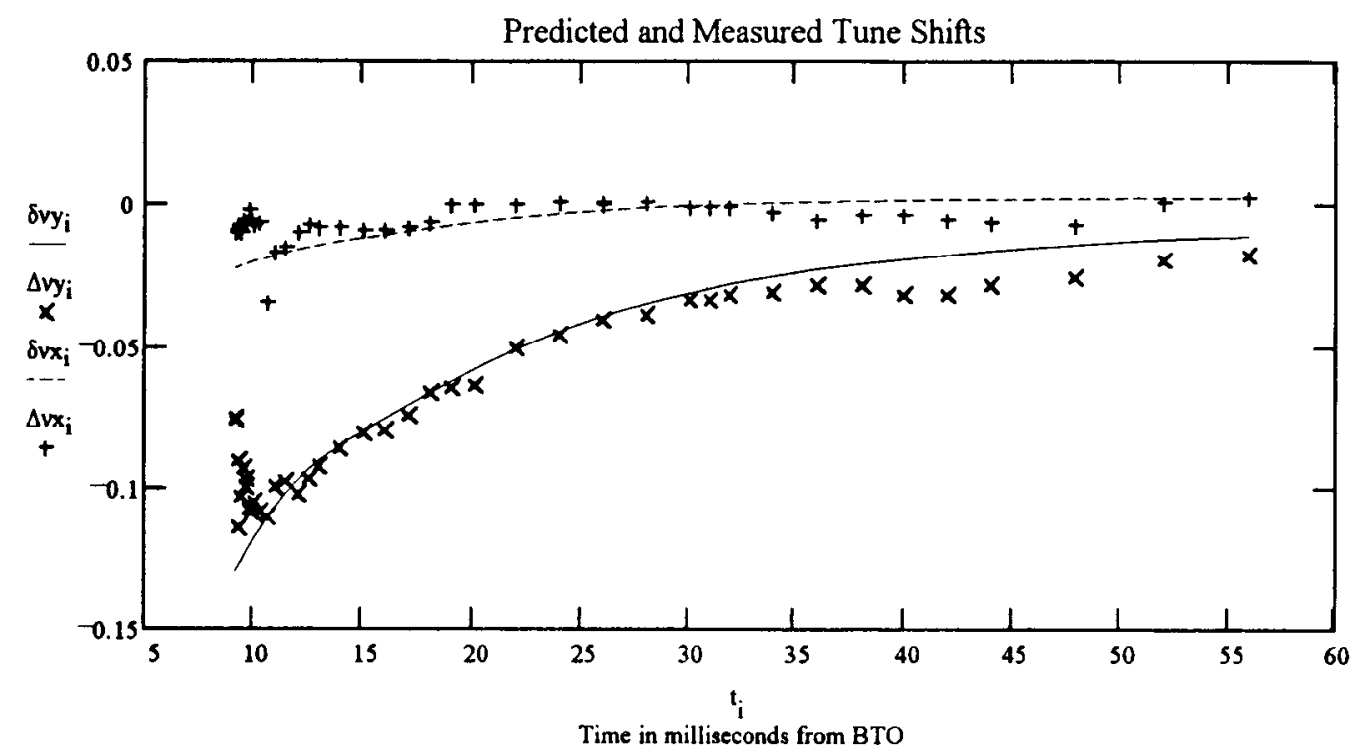

Graph 7: Predicted versus measured tune shifts. +'s are measured horizontal shift, x's are measured vertical shift, dashed line is predicted horizontal shift, solid line is predicted vertical shift.

\section{CONCLUSION:}

The data and theory agree reasonably well. However, given the uncertainty in the value of the peak line charge density, amongst other things, the good agreement may be misleading. A measurement of the coherent tune shift has been done earlier with good agreement and measured peak line density (bunching factor). ${ }^{10}$

There were radial shifts and non-zero chromaticites throughout the cycle. This may account for some of the differences between predicted and measured values. If these measurements are taken again, the radius should be held as constant as possible. The chromaticity should also be constant throughout the cycle. The uncertainty in the peak line charge density is potentially the source of the greatest error. This can be measured rather directly if the measurements are done again. The Band III RF cavities were on during the $\mathrm{Rf}$ capture part of the cycle. This might reduce the peak line charge density

The vertical tune shift increases to its maximum value about $1 \mathrm{~ms}$ after injection begins. The injection process is $500 \mu \mathrm{s}$ long. The intensity increases during this process. Since the tune shift is intensity dependent, it is not be surprising to find the shift increasing during injection. The overall momentum and peak charge density may be changing during and shortly after this time as the $\mathrm{Rf}$ captures the injected beam. These factors may also contribute to the increasing shift during and just after injection. Also, as was noted earlier, during these early measurements some beam may have been kicked out. This may affect the tune shift. The graph on the next page shows the early behavior of the tune shifts.

One might expect that very early in injection the tune in the vertical would match the tune given by the BoosterTuneControl application. The measurements suggest that it is considerably lower than that. It is not 
clear why the prediction of the application is so different from what is measured during this part of the cycle. It might be interesting to measure the tune at injection, with low intensity, when it is set to be close to 5 .

The daily injection tune measurement using PIP generally indicates a tune of about 4.98 for this setting..$^{9}$ The PIP measurement is done with only half a turn of beam, so space charge effects should be insignificant.

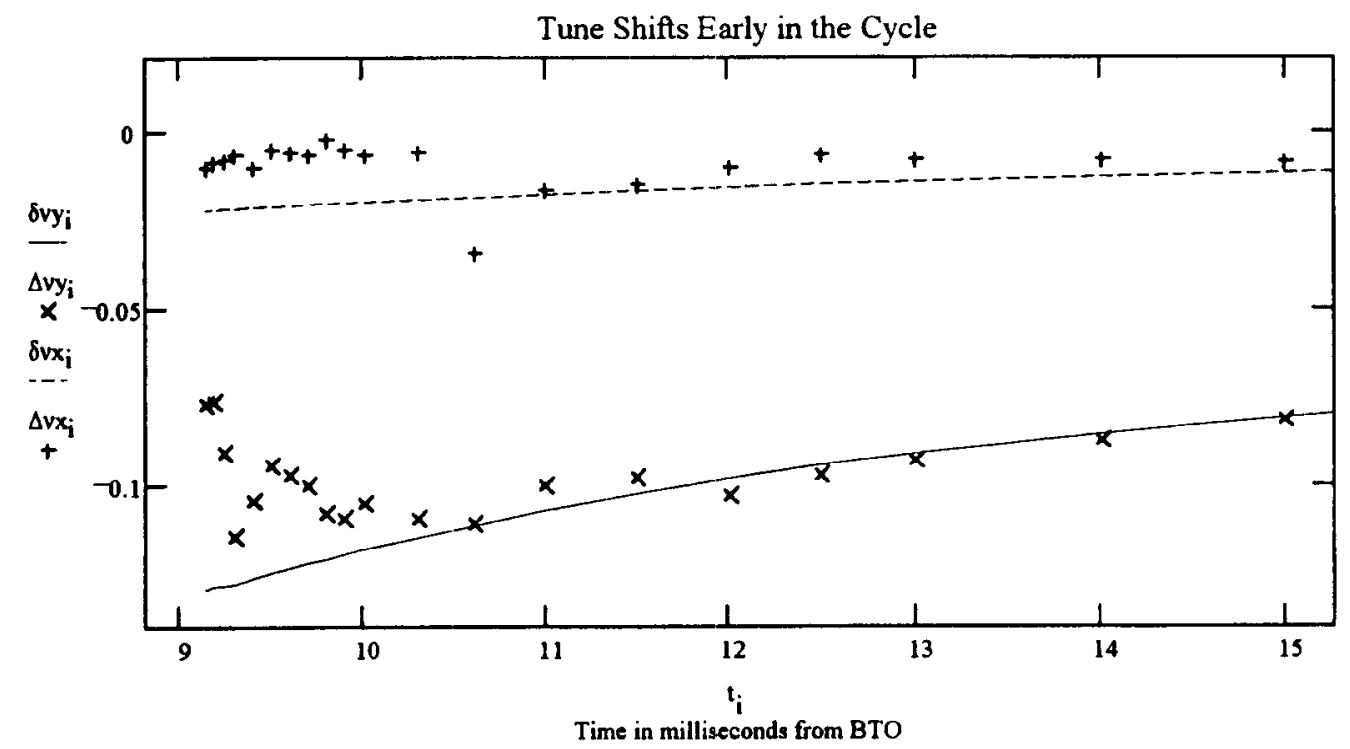

Graph 8: Predicted and measured tunes during the first few milliseconds of the cycle.

+'s are measured horizontal shift.

$x$ 's are measured vertical shift.

dashed line is predicted horizontal shift.

solid line is predicted vertical shift. 


\section{Appendix:}

\section{Estimating the Incoherent Tune Spread}

The incoherent tune spread is of more practical interest than the coherent tune shift. Using the machinery developed for the above analysis, a few other measurements, and results from the MAD model of the Booster and BTA, an estimate of the incoherent tune spread can be made relatively easily.

The expressions for the maximum incoherent tune shift are of the form, 2,6

$$
\delta v_{\text {incoh }}=\frac{-\mathrm{r} \cdot \mathrm{R}^{2}}{V \cdot \beta^{2} \cdot \gamma} \cdot\left(\frac{1}{\gamma^{2}} \cdot \mathrm{F}_{\text {incoh }}+\beta^{2} \cdot G\right) \cdot
$$

The $\mathrm{F}_{\text {incoh }}$ term is dependent on the beam size, it is given by ${ }^{2}$,

$$
\begin{aligned}
& \mathrm{Fy}_{\text {incoh }_{i}}=\left[\frac{1}{\left(a_{i}\right)^{2}}+\frac{\mathrm{fr}}{\mathrm{adc}^{2}}\right] \cdot p \lambda_{i} \\
& \mathrm{Fx}_{\text {incoh }_{i}}=\left[\frac{1}{\left(a_{i}\right)^{2}}-\frac{\mathrm{fr}}{\mathrm{adc}^{2}}\right] \cdot p \lambda_{i}
\end{aligned}
$$

' $a$ ' is related to the beam size. The field caused by image charges on a circular vacuum chamber vanish if the beam has a circular charge distribution. ${ }^{16}$ The beam is treated as though it has a circular charge distribution. However, the vacuum chamber is elliptical inside the dipoles, and so, the field is not zero there. This is the reason for the second term, $\mathrm{fr} / \mathrm{a}_{\mathrm{dc}}{ }^{2}$. The image charges can be treated as if there are no charges present in the vacuum chamber. Then, the field produced by the image charges follows Laplace's equation $\left(\partial \mathrm{E}_{x} \partial \mathrm{x}=-\partial \mathrm{E}_{\mathrm{y}} \partial \mathrm{y}\right){ }^{11}$ Hence, within the dipoles the asymmetrical vacuum chamber causes an image electric field which defocuses the beam vertically and focuses it horizontally by the same amount.

Finding the Beam Size and the parameter 'a':

Since the beam size inside the Booster is a function of longitudinal position, the emittance multiplied by the average Beta function is used to approximate it. An estimate for the emittance can be obtained from the beam size on the multiwire at $6 \mathrm{ft}$. in BTA. The beam size on the multiwire was measured within a few hours after the study at similar intensity ( $79.8 \times 10^{12}$ for 4 cycles). A MAD run using nominal values of the optical functions and dispersion at the beginning of BTA predicts that $\beta_{x}=4.09 \mathrm{~m}, \beta_{y}=17.86 \mathrm{~m}$, and $D_{x}=0.61 \mathrm{~m}^{3}$ The measured beam size was $8.88 \mathrm{~mm}$ FWHM in the horizontal, and $19.20 \mathrm{~mm}$ FWHM in the vertical. ${ }^{4}$ Assuming the beam is gaussian in shape this gives, 
For the Vertical Plane:

FWHM $_{y}=19.20 \quad \beta_{y}:=17.86$

Solving $0.5=\exp \left(-\frac{F W H M_{y}^{2}}{2 \cdot \sigma y^{2}}\right)$ yields $\sigma y:=15.2 \mathrm{~mm}$ for the vertical plane.

$\varepsilon_{y}:=\frac{(\sigma y)^{2}}{\beta_{y}} \quad$ gives $\varepsilon_{y}=12.936 \pi \mathrm{mm} \mathrm{mrad}$.

The beam size in the horizontal plane is affected by the momentum spread. What is $\Delta \mathrm{p} / \mathrm{p}$ at extraction? The momentum spread throughout the cycle and at extraction can be found using longitudinal parameters,

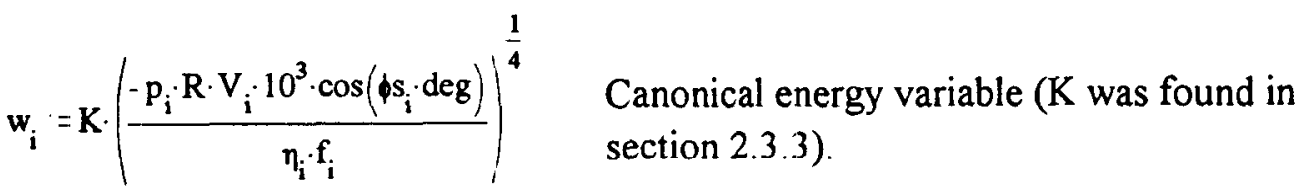

$$
\begin{aligned}
& \mathrm{E}_{\mathrm{i}}=\sqrt{\left(\mathrm{p}_{\mathrm{i}}\right)^{2}+(\mathrm{m})^{2}} \quad \text { Total Energy. } \\
& p_{\text {spread }_{i}}=\frac{2 \cdot f_{i} \cdot w_{i}}{E_{i} \cdot 10^{9} \cdot\left(\beta_{i}\right)^{2}} \quad \text { Momentum spread. }{ }^{7}
\end{aligned}
$$

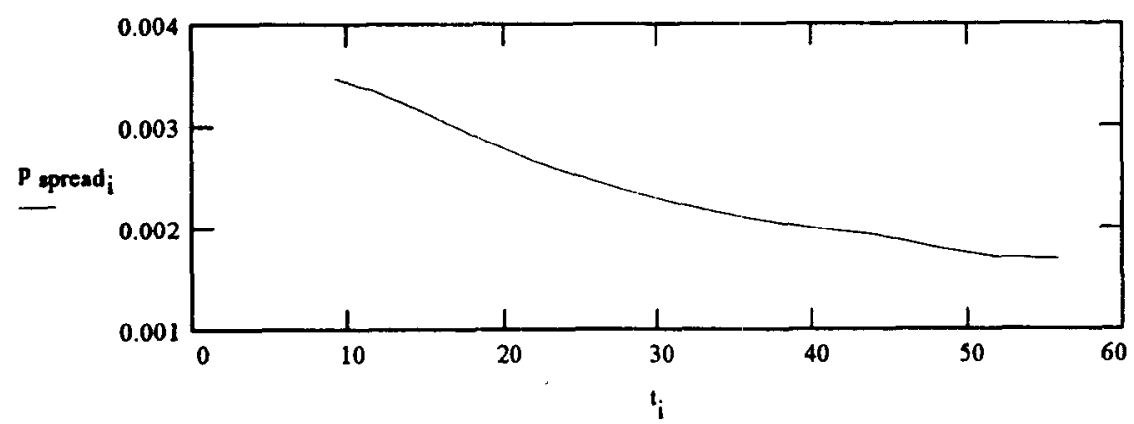

Graph 9: Calculated momentum spread through cycle.

This same analysis at extraction momentum $(67 \mathrm{~ms})$ gives:

$$
\text { pspread }_{\text {ext }}=1.7 \cdot 10^{-3} \quad(\Delta \mathrm{p} / \mathrm{p} \text { at extraction })
$$

$B_{\text {ext }}=5551$ field at extraction [gauss]. ${ }^{9}$

$$
\text { FWHM }_{X}=8.88 \quad \mathrm{D}=0.61 \quad \beta_{\mathrm{X}}=4.09
$$


Solving $0.5=\exp \left(-\frac{F W H M_{X}{ }^{2}}{2 \cdot \sigma x^{2}}\right)$ yields $\sigma x=7.54 \mathrm{~mm}$ for the horizontal plane.

$\sigma x^{2}=\beta_{x} \varepsilon_{x}+\left(D \cdot p^{2 p r e a d} \text { ext } 1000\right)^{2}$ yields $\quad \varepsilon_{x}=\frac{\left(\sigma x^{2}-1000000 \cdot D^{2} \cdot \text { pspread }^{2}{ }^{2}\right)}{\beta_{x}}$

So, $\varepsilon_{X}=13.637 \pi \mathrm{mm} \mathrm{mrad}$, this is close to the emittance in the y plane $(12.9 \pi \mathrm{mm}$ mrad). For simplicity the emittances in both planes are assumed equal throughout the cycle.

$\varepsilon_{i}=\varepsilon_{y} \cdot \frac{B}{B_{i}} \quad$ Calculating emittance assuming adiabatic damping throughout the cycle $[\pi \mathrm{mm} \mathrm{mrad}]$.

The beam dimensions vary around the Booster ring. As an approximation the $\beta_{\mathrm{av}}=8.0 \quad \mathrm{D}_{\mathrm{av}}=1.6 \quad$ average dimensions, or the size when $\beta$ and $\mathrm{D}$ are their average values can be used. ${ }^{16}$ The average value of $\beta$ is almost the same in both planes $\left(\beta_{x}=7.9 \mathrm{~m}\right.$, $\beta_{\mathrm{y}}=8.0 \mathrm{~m}$ ). These values are from the MAD model for the Booster.

$\sigma_{\mathrm{i}}=\frac{\sqrt{\beta_{\mathrm{av}} \varepsilon_{\mathrm{i}}}}{1000} \quad$ Booster beam size neglecting dispersion.

$\sigma_{\text {total }_{i}}=\frac{\sqrt{\beta_{\text {av }} \varepsilon_{i}+\left(D_{\text {av }} \cdot p_{\text {spread }} \cdot 1000\right)^{2}}}{1000} \quad$ Booster beam size including dispersion.

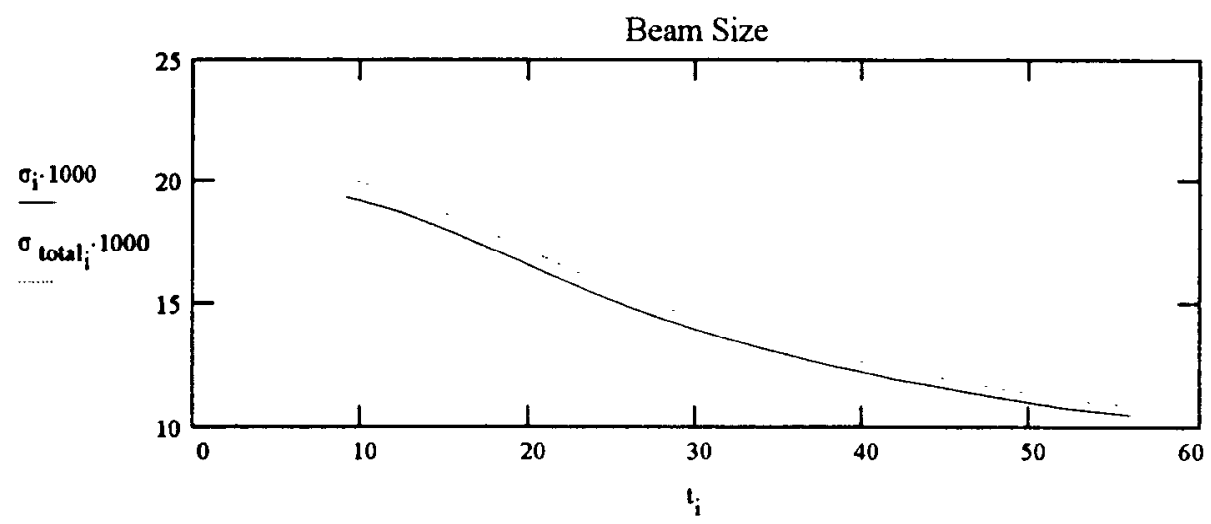

Graph 10: One $\sigma$ Beam size with (dashed) and without (solid) dispersion [mm].

The graph above shows that the effect of dispersion is relatively small. Therefore, it will be neglected.

The expression for the maximum direct space charge tune shift that will be used assumes that the beam's cross section is round. The above analysis suggests that this is a reasonable approximation. It also treats the distribution inside a radius ' $a$ ' as uniform. ${ }^{6}$ The distribution is probably more Gaussian than uniform. The normalized density at the center of a gaussian is $1 / 2 \pi \sigma^{2}$. The approximation uses this as the normalized density of the uniform distribution of area $\pi \mathrm{a}^{2}$. 
Setting two expressions for density equal to each other, $\quad \frac{1}{\pi \cdot a^{2}}=\frac{1}{2 \cdot \pi \cdot \sigma^{2}} \quad$ which is equivalent to $\frac{1}{a^{2}}=\frac{1}{2 \cdot \sigma^{2}}$
and then solving for ' $a$ '.

$a_{i}=\sqrt{2} \cdot \sigma \quad$ so, ' $a$ ' is the equivalent radius for a uniform distribution with a density equal to that at the center of a gaussian.

Finding the Maximum and Minimum Incoherent Tune Shifts:

$$
\begin{gathered}
\text { Fy }_{\text {incoh }_{i}}=\left[\frac{1}{\left(a_{i}\right)^{2}}+\frac{f r}{a_{d c}^{2}}\right] \cdot p \lambda_{i} \quad F x_{\text {incoh }_{i}}=\left[\frac{1}{\left(a_{i}\right)^{2}}-\frac{f r}{a_{d c}^{2}}\right] \cdot p \lambda_{i} \\
\delta v y_{\text {incoh }_{i}}=\frac{-r_{0} \cdot R^{2}}{v_{i, 1} \cdot\left(\beta_{i}\right)^{2} \cdot \gamma_{i}} \cdot\left[\frac{1}{\left(\gamma_{i}\right)^{2}} \cdot F y_{\text {incoh }_{i}}+\left(\beta_{i}\right)^{2} \cdot G y_{i}\right] \\
\delta v x_{\text {incoh }_{i}}=\frac{-r_{o} \cdot R^{2}}{v_{i, 0} \cdot\left(\beta_{i}\right)^{2} \cdot \gamma_{i}} \cdot\left[\frac{1}{\left(\gamma_{i}\right)^{2}} \cdot F x_{\text {incoh }_{i}}+\left(\beta_{i}\right)^{2} \cdot G x_{i}\right]
\end{gathered}
$$

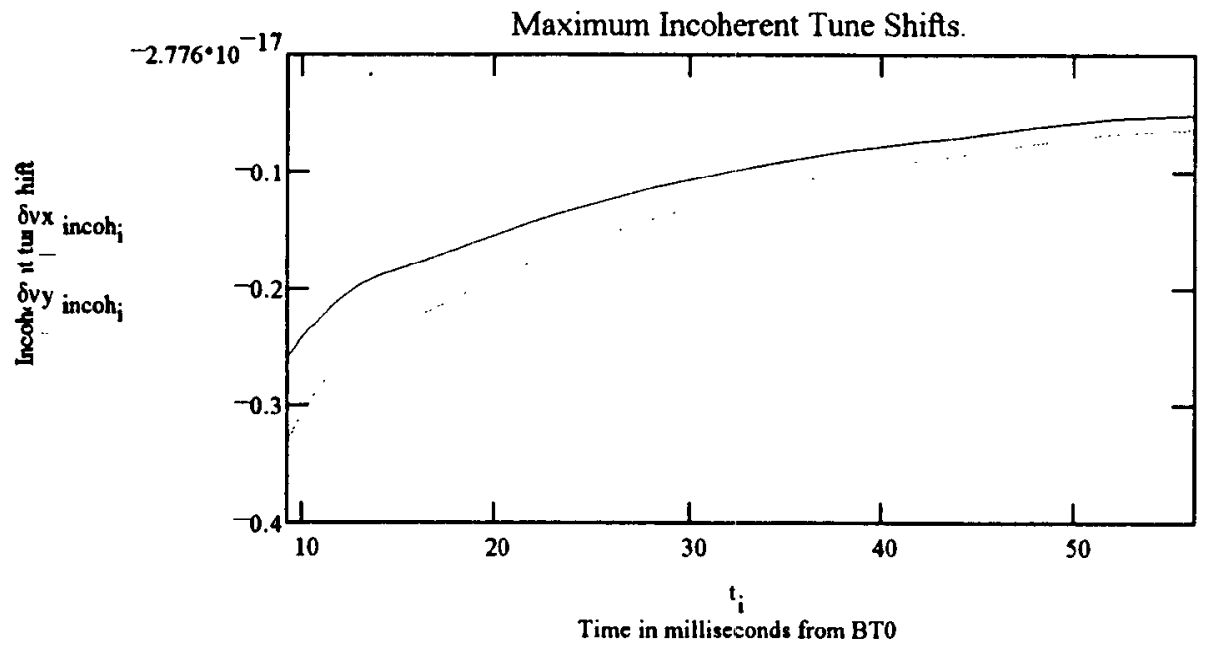

Graph 11: Maximum incoherent tune shifts in vertical (dotted) and horizontal (solid) planes.

The minimum tune shifts occur when the particle is at the longitudinal edge of the beam. The beam charge density there is relatively close to zero. So, those particles are only affected by the dc components of the fields. 
$\delta_{\text {vymin }}$ incoh $_{i}=\frac{-r_{0} \cdot R^{2}}{v_{i, 1} \cdot\left(\beta_{i}\right)^{2} \cdot y_{i}} \cdot\left[\left(\beta_{i}\right)^{2} \cdot G y_{i}\right]$

$\delta_{v x \sin _{\text {incoh }}}:=\frac{\mathrm{r}_{0} \cdot \mathrm{R}^{2}}{v_{i, 0} \cdot\left(\beta_{i}\right)^{2} \cdot y_{i}} \cdot\left[\left(\beta_{i}\right)^{2} \cdot G x_{i}\right]$

Minimum incoherent tune shifts.

These are the maximum and minimum possible tunes in the $\mathrm{x}$ and $\mathrm{y}$ planes.

$$
\begin{array}{ll}
v_{y_{\max }}:=v_{i, 1}+\delta v y \min \text { incoh }_{i} & v_{x_{\max }}=v f_{i, 0}+\delta v x \sin \text { incoh }_{i} \\
v_{y \operatorname{ymin}_{i}}:=v_{i, 1}+\delta v y_{\text {incoh }} & v_{x_{i} \min _{i}}=v_{i, 0}+\delta v x_{\text {incoh }_{i}}
\end{array}
$$

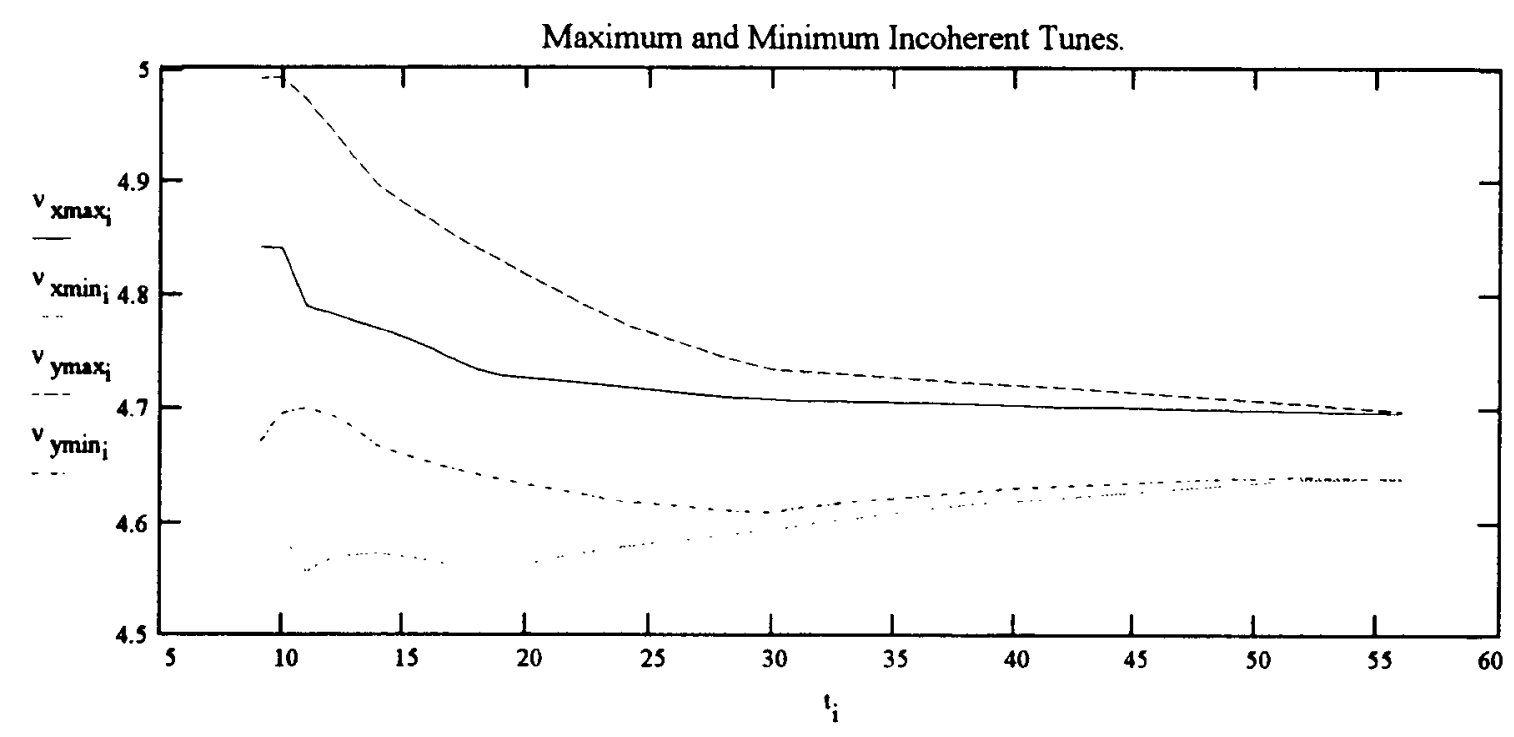

Graph 12: Maximum and minimum incoherent tunes. These are the BoosterTuneControl functions plus the incoherent tune shifts (max. and $\min$.).

Top trace is with minimum vertical shift.

Second trace from the top is with minimum horizontal shift.

Third trace from the top is with maximum vertical shift.

Bottom trace is with maximum horizontal shift. 
DATA: ${ }^{12,13,14,15}$

\begin{tabular}{|c|c|c|c|c|c|c|c|}
\hline $\begin{array}{l}\text { Time from } \\
\text { BTO (ms) }\end{array}$ & $\begin{array}{l}\text { Meas. } \\
\text { Tune }(H)\end{array}$ & $\begin{array}{l}\text { Calc. } \\
\text { Tune }(\mathrm{H})\end{array}$ & $\begin{array}{l}\text { Meas. Tune } \\
\text { (V) }\end{array}$ & $\begin{array}{l}\text { Calc. Tune } \\
\text { (V) }\end{array}$ & $\begin{array}{l}\text { B field } \\
\text { (gauss) }\end{array}$ & $\begin{array}{l}\text { Rf Band II } \\
\text { Voltage } \\
\text { (kV) }\end{array}$ & $\begin{array}{l}\text { Synchronous } \\
\text { phase (degrees) }\end{array}$ \\
\hline 9.15 & 4.82 & 4.83 & 4.923 & 5.000 & 1539.7 & 90 & 5.3 \\
\hline 9.2 & 4.821 & 4.83 & 4.924 & 5.000 & 1541.4 & 90 & 5.3 \\
\hline 9.25 & 4.822 & 4.83 & 4.909 & 5.000 & 1542.8 & 90 & 5.3 \\
\hline 9.3 & 4.823 & 4.83 & 4.886 & 5.000 & 1544.2 & 90 & 5.3 \\
\hline 9.4 & 4.82 & 4.83 & 4.896 & 5.000 & 1547.4 & 90 & 5.3 \\
\hline 9.5 & 4.825 & 4.83 & 4.906 & 5.000 & 1550.4 & 90 & 5.3 \\
\hline 9.6 & 4.824 & 4.83 & 4.903 & 5.000 & 1553.4 & 90 & 5.3 \\
\hline 9.7 & 4.823 & 4.83 & 4.9 & 5.000 & 1556.4 & 90 & 5.3 \\
\hline 9.8 & 4.828 & 4.83 & 4.8924 & 5.000 & 1559.4 & 90 & 5.3 \\
\hline 9.9 & 4.825 & 4.83 & 4.891 & 5.000 & 1562.4 & 90 & 5.3 \\
\hline 10 & 4.823 & 4.83 & 4.895 & 5.000 & 1565.4 & 90 & 5.3 \\
\hline 10.3 & 4.809 & 4.815 & 4.885 & 4.994 & 1574.2 & 90 & 5.3 \\
\hline 10.6 & 4.765 & 4.8 & 4.877 & 4.988 & 1583.4 & 90 & 5.3 \\
\hline 11 & 4.763 & 4.78 & 4.88 & 4.980 & 1595.2 & 90 & 5.3 \\
\hline 11.5 & 4.762 & 4.777 & 4.869 & 4.967 & 1611.5 & 90 & 5.9 \\
\hline 12 & 4.764 & 4.774 & 4.852 & 4.955 & 1631 & 90 & 6.5 \\
\hline 12.5 & 4.764 & 4.771 & 4.845 & 4.942 & 1650.5 & 90 & 7 \\
\hline 13 & 4.759 & 4.767 & 4.836 & 4.929 & 1672.1 & 90 & 7.7 \\
\hline 14 & 4.753 & 4.761 & 4.817 & 4.904 & 1719.4 & 90 & 8.7 \\
\hline 15 & 4.744 & 4.753 & 4.808 & 4.889 & 1772.8 & 90 & 9.7 \\
\hline 16 & 4.735 & 4.744 & 4.795 & 4.875 & 1829.4 & 90 & 10.6 \\
\hline 17 & 4.726 & 4.734 & 4.786 & 4.861 & 1891 & 90 & 11.5 \\
\hline 18 & 4.719 & 4.725 & 4.781 & 4.848 & 1957.9 & 90 & 12.3 \\
\hline 19 & 4.719 & 4.719 & 4.771 & 4.826 & 2028.2 & 90 & 13 \\
\hline 20 & 4.717 & 4.717 & 4.76 & 4.824 & 2101.4 & 90 & 13.6 \\
\hline 22 & 4.714 & 4.714 & 4.751 & 4.802 & 2260.7 & 90 & 14.6 \\
\hline
\end{tabular}




\begin{tabular}{|l|l|l|l|l|l|l|l|}
\hline $\begin{array}{l}\text { Time from } \\
\text { BTO (ms) }\end{array}$ & $\begin{array}{l}\text { Meas. } \\
\text { Tune }(\mathrm{H})\end{array}$ & $\begin{array}{l}\text { Calc. } \\
\text { Tune }(\mathrm{H})\end{array}$ & $\begin{array}{l}\text { Meas. Tune } \\
(\mathrm{V})\end{array}$ & $\begin{array}{l}\text { Calc. Tune } \\
(\mathrm{V})\end{array}$ & $\begin{array}{l}\text { B field } \\
(\text { gauss })\end{array}$ & $\begin{array}{l}\text { Rf Band II } \\
\text { Voltage } \\
(\mathrm{kV})\end{array}$ & $\begin{array}{l}\text { Synchronous } \\
\text { phase (degrees) }\end{array}$ \\
\hline 24 & 4.711 & 4.710 & 4.734 & 4.780 & 2427.6 & 90 & 15.4 \\
\hline 26 & 4.707 & 4.706 & 4.724 & 4.765 & 2599.7 & 90 & 15.8 \\
\hline 26 & 4.706 & 4.706 & 4.724 & 4.765 & 2599.7 & 90 & 15.8 \\
\hline 28 & 4.703 & 4.702 & 4.711 & 4.750 & 2778.6 & 90 & 15.8 \\
\hline 30 & 4.699 & 4.7 & 4.705 & 4.739 & 2955.4 & 90 & 15.8 \\
\hline 31 & 4.698 & 4.699 & 4.704 & 4.738 & 3045.8 & 89.3 & 16 \\
\hline 32 & 4.698 & 4.699 & 4.704 & 4.736 & 3134.2 & 88.7 & 16.2 \\
\hline 34 & 4.695 & 4.698 & 4.702 & 4.733 & 3311.4 & 87.3 & 16.4 \\
\hline 36 & 4.693 & 4.698 & 4.701 & 4.730 & 3490.3 & 86 & 16.6 \\
\hline 38 & 4.693 & 4.697 & 4.698 & 4.727 & 3669.1 & 84.7 & 17 \\
\hline 40 & 4.692 & 4.696 & 4.693 & 4.725 & 3845.1 & 83.3 & 17.2 \\
\hline 42 & 4.69 & 4.695 & 4.69 & 4.722 & 4024.2 & 82 & 17.5 \\
\hline 44 & 4.689 & 4.695 & 4.69 & 4.719 & 4203.4 & 80.7 & 17.8 \\
\hline 48 & 4.686 & 4.693 & 4.687 & 4.713 & 4557.3 & 69.8 & 20.7 \\
\hline 52 & 4.693 & 4.692 & 4.687 & 4.707 & 49126 & 61.8 & 23.6 \\
\hline 56 & 4.693 & 4.69 & 4.683 & 4.701 & 5194.6 & 60 & 14.2 \\
\hline
\end{tabular}

\section{Acknowledgements:}

I thank Mike Blaskiewicz for his help and encouragement.

\section{References:}

[1]. W. van Asselt, BoosterTuneControl application transfer functions, private communication.

[2]. M. Blaskiewicz, space charge tune shift expressions, private communication.

[3]. P. Sampson, MAD model for BTA, private communication.

[4]. J. Laster, Morning Numbers for May 4, 1995.

[5]. HEP Startup Book III FY'95. pg. 136.

[6]. A.W. Chao, The Physics of Collective Beam Instabilites in High Energy Accelerators. 
Wiley. 1993.

[7]. W.T. Weng and S.R. Mane, Fundamentals of Particle Beam Dynamics and Phase Space. Pg. 37-42, September 4, 1991. AGS Dept.

[8]. Y.Y. Lee, R. Thomas, Booster Design Manual, Booster Parameter List, pg.1-10, 1988.

[9]. Daily Morning Numbers for FY'95.

[10]. W. van Asselt, Measurement of Coherent Space Charge Shifts in the Booster, AGS Studies Report No. 304, April 4, 1993.

[11]. B. W. Zotter, Tune Shifts of Excentric Beams in Elliptic Vacuum Chambers, IEEE Transactions on Nuclear Science, NS-22, pg. 1451, 1975.

[12]. HEP Startup Book III FY'95. pgs. 106-108.

[13]. BoosterTuneControl Application archive "B1_for2_5_4_95".

[14]. BoosterMainMagnet Application archive "B1_for2_5_4_95".

[15]. BoosterRfBeamControl Application archive "B1_for2_5_4_95".

[16]. G. Parzen and K. Jellett, The Effect of the Beam Self-field on the Transverse Betatron Oscillation Frequency, IXth Intern. Conf. High-Energy Accelerators, May 2-7, 1974, SLAC, Stanford, $\mathrm{Ca}$. 